\title{
Development of Data Models for Nursing Assess- ment of Cancer Survivors Using Concept Analysis
}

\author{
Myung Kyung Lee, RN, MPH, Hyeoun-Ae Park, RN, PhD \\ College of Nursing, Seoul National University, Seoul, Korea
}

Objectives: Sharing of cancer-related information among healthcare professionals is crucial to ensuring the quality of longterm care for cancer survivors. Appropriate distribution of the essential facts can be achieved using data models. The purpose of this study was to develop and validate suitable data models for use in the nursing assessment of cancer survivors. Methods: The models developed in this study were based on a modification of concept analysis developed by Walker and Avant. Our approach involved determining the purpose of the analysis, identifying data elements, defining these elements and their uses, determining critical attributes, value sets, and cardinalities, and ultimately constructing data models which were examined externally by domain experts. Results: We developed 112 data models with 112 data elements, 29 critical attributes, 102 value sets, and 6 data types for the assessment of cancer survivors. External validation revealed that the data elements, critical attributes, and value sets proposed were comprehensive, relevant, and sufficiently useful to encompass nursing issues related to cancer survivors. Conclusions: Data models developed in this study will contribute to ensuring the semantic consistency of data collected from cancer survivors, which will improve the quality of nursing assessments and in turn translate to improved long-term patient care.

Keywords: Standards of Care, Nursing Assessment, Quality of Healthcare, Electronic Health Record

\section{Introduction}

There are approximately 28 million cancer survivors living with cancer worldwide [1]. The number of cancer survivors will increase steadily in the coming years as the average age of the world's population increases. A cancer survivor is de-

Received for review: February 7, 2011

Accepted for publication: March 14, 2011

\section{Corresponding Author}

Hyeoun-Ae Park, RN, PhD

College of Nursing, Seoul National University, 28 Yeongeon-dong, Jongno-gu, Seoul 110-799, Korea. Tel: +82-2-740-8827, Fax: +822-765-4103, E-mail: hapark@snu.ac.kr

This is an Open Access article distributed under the terms of the Creative Commons Attribution Non-Commercial License (http://creativecommons.org/licenses/by$\mathrm{nc} / 3.0 /$ ) which permits unrestricted non-commercial use, distribution, and reproduction in any medium, provided the original work is properly cited.

(c) 2011 The Korean Society of Medical Informatics fined as anyone who has been diagnosed, is living with, or has recovered from cancer, and family members who are affected by a diagnosis of cancer [2].

There is a growing need to improve the physical, social, psychological, and spiritual well-being of cancer survivors during the course of deciding treatment options, enduring treatments, and surviving the disease $[2,3]$. The data elements required to assess the physical, social, psychological, and spiritual well-being of cancer survivors need to be continuously tracked and integrated into the cancer survivor's follow-up care, even years after becoming cancer free $[4,5]$.

The sharing of cancer-related information among healthcare professionals is key to the quality of long-term cancer care for cancer survivors [5]. The cancer-related information can only be shared if they are represented in a way that all healthcare professionals can understand [6]. The ability to exchange clinical data between different computer systems and maintain data consistently in a longitudinal electronic recording system is also important for ensuring the qual- 
ity of long-term cancer care [5]. The ability of all healthcare professionals and multiple systems to understand the clinical data is known as semantic interoperability. One way to ensure semantic interoperability is to model data by specifying the following [7]:

- What are the key data elements?

- What are the critical attributes?

- What is possible value set for each attribute?

- Are the attributes optional or mandatory?

- What other rules need to be expressed?

Ongoing research on data modeling has been underway in various countries including Australia, the Netherlands, USA, and Korea, and through international standard development organizations such as health level seven (HL7). The names for these models include the openEHR archetype in Australia [8], the detailed clinical model in the netherlands, HL7 [9], clinical element models in the USA, the intermountain healthcare [10], and the clinical contents model in Korea [11]. However, these works have been limited to the medical domain [8-11]. Although nurses and physicians sometimes handle the same situations, they often view these situations in different ways, and so data models for the medical domain cannot be used in the nursing domain. It has thus been emphasized over the past few years that the nursing profession should develop its own data models [7].

The benefits of data models are that they allow an accurate correspondence of clinical data in a consistent, safe, and meaningful way, and they can adapt to the changing information needs of different healthcare professions and institutions [12]. As an increasing number of people are affected by cancer, and various workforces participate in cancer management, a data model is needed to collect and share clinical data to enable improvements in the quality and efficiency of cancer care. The purpose of this study was thus to develop and validate data models for the nursing assessment of cancer survivors using concept analysis.

\section{Methods}

The development of the data models was guided by a modification of the concept analysis developed by Walker and Avant [13], which was chosen because its process [14-16] is well suited for the development of data models (Figure 1).

The concept analysis of Walker and Avant comprises the following steps [13]: 1) identifying the concept, 2) determining the purposes of the analysis, 3) defining the concept and its uses, 4) determining the critical attributes, 5) constructing the cases, 6) identifying the antecedents and conse-
Walker and Avant's concept analysis

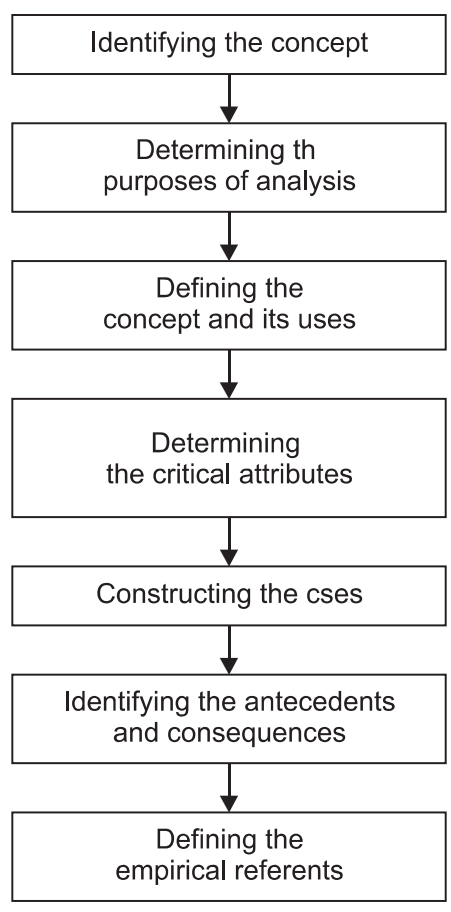

Data model development

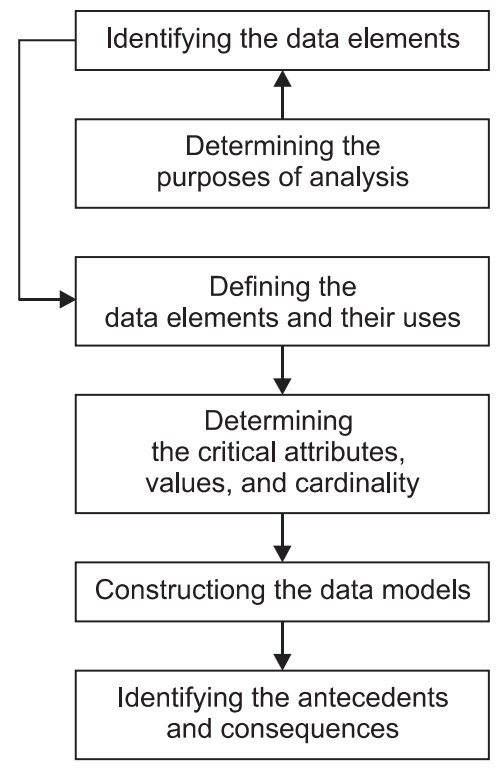

We determined the purpose of the analysis before we identified any concept, because we analyzed more than one concept with a particular purpose.

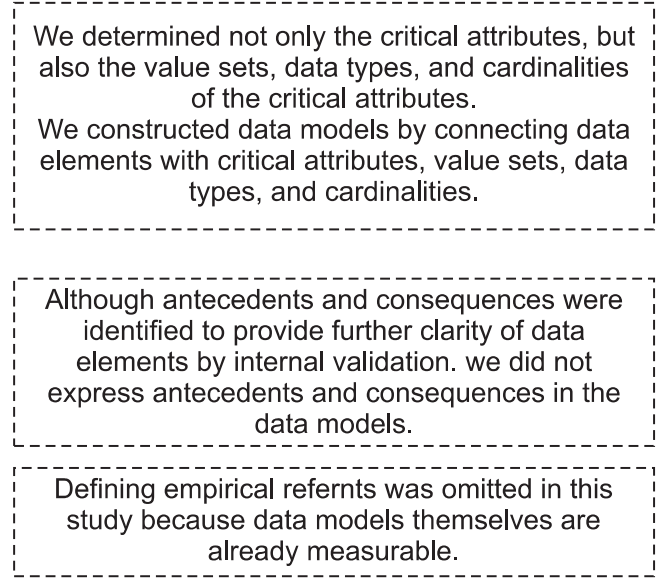
sets, data types, and cardinalities ted data models by connecting data types, and cardinalities

Figure 1. The process of Walker and Avant's concept analysis and its modification for data model development. 
quences, and 7) defining the empirical referents. The concept analysis usually has a single purpose, such as the generation of a theoretical model or of a measurement instrument for a particular concept of interest. Thus, identifying a concept is followed by determining its purpose. However, for developing the data model, we determined the purpose of the analysis before we identified any data element, because we analyzed more than one data element with a particular purpose, such as exchanging and sharing clinical data in an electronic health record (EHR) system. Since we dealt with more than one data element, we defined multiple data elements in this study, and their uses in step 3. In step 4 we determined not only the critical attributes, but also the value sets, data types, and cardinalities of the critical attributes. In step 5 we constructed data models by connecting data elements with critical attributes, value sets, data types, and cardinalities. In step 6 we identified antecedents and consequences to provide further clarity of the data elements by internal validation. However, we did not express antecedents and consequences in the data models. Step 7, defining the empirical referents, was omitted in this study because the data models themselves were already measurable, having specified critical attributes with value sets, data types, and cardinalities.

\section{Determining the Purposes of the Analysis}

The purpose of this analysis was to develop data models for the nursing assessment of cancer survivors that will enable the collection and sharing of clinical data among healthcare professionals and between healthcare institutions.

\section{Identifying the Data Elements}

Data elements for the nursing assessment of cancer survivors were identified from clinical nursing statements used to describe sign and symptom, and nursing diagnosis in the electronic nursing records of cancer patients who were hospitalized or visited outpatient department in a tertiary hospital by extracting key concepts. For example, we extracted the key concept "discomfort" from the following statements: "discomfort is present," "decreased pharyngolarynx discomfort," "complains of discomfort," and "no discomfort after eating." In addition, we reviewed the medical or nursing dictionary, nursing literature such as textbooks, and nursing and medical articles on oncology, nursing terminology classifications such as international nursing diagnosis and the international classification for nursing practice (ICNP), and consulted nurse experts to supplement the data elements. The extracted data elements were classified into physical, psychological, and spiritual domains based on previous research [2].

\section{Defining the Data Elements and Their Uses}

Data elements were defined to clarify what we mean when referring to text definitions of concepts in the ICNP, or formal definitions of concepts from the systematized nomenclature of medicine-clinical term. We also used medical and nursing dictionaries to obtain definitions of the data elements. We identified the uses of the data elements utilized in nursing practice by reviewing nursing forms, nursing statements, and the research published in nursing and medical articles.

\section{Determining the Critical Attributes, Value Sets, and Cardinalities}

We determined the critical attributes of data elements, which are qualifiers or modifiers to represent data elements in more detail, by reviewing nursing statements, nursing forms, and the relevant literature. We then identified possible value sets for critical attributes by referring to nursing statements and the relevant literature. The cardinality of each attribute was determined. Nurse experts participated in determining the critical attributes, value sets, and cardinalities. Finally, the data type of each attribute was classified based on the HL7 data type list [17], such as "Integer (INT)," "String (ST)," "Physical Quantity (PQ)," and "Ratios (RTO)" [18].

\section{Constructing Data Models}

We constructed data models by linking each data element with critical attributes, value sets, data types, and cardinalities. We present each data model in table form.

\section{Validation of Data Models}

Two nursing terminology experts and nine nurse informaticists reviewed the process of data model development as well as the data model themselves, and they also reviewed the clarity of the data models by identifying antecedents and consequences. The two nursing terminology experts have been engaged in teaching and research related to the terminology for more than 10 years, and the nine nursing informatists have had more than 5 years of experience as clinical nurses, three of them in internal medicine units, three of them in surgical units, and three in oncology nursing units. Currently, four of them work as nurse informaticists at an EMR center in a tertiary hospital. Five of them have doctoral degree in nursing informatics and four have master's degrees in the same.

In addition, an expert panel of eleven clinicians comprising four oncology nurses, five nurse researchers, one clinical doctor, and one social worker verified the face validity of the data models. The members of the expert panel have worked 
in oncology for at least five years. Six of them have doctoral degrees and five have master's degrees. The questions used to check the face validity were developed based on the criteria published for evaluating the content and modeling structure of health terminology in previous studies, such as "usefulness" [19], "reusability" [19,20], "non-ambiguity" [2123], "comprehensiveness" [19,21-23], "non-redundancy" $[19,21,22]$, and clinical relevancy" [19]. During this external validation, 11 items-questions on clinical relevancy, usefulness, reusability, non-ambiguity, comprehensiveness, and non-redundancy were asked, and the responses to which were scored on a 5-point Likert scale (from $1=$ strongly agree to $5=$ strongly disagree). We presented data distribution in a frequency.

\section{Results}

\section{Identifying and Defining the Data Elements}

A total of clinical nursing statements of 98 patients whose care time ranged 2 days to 20 months since surgery were analyzed to extract data elements. The mean patients' age was 51 years $( \pm 5.5)$ and fifty three patients $(54.1 \%)$ were male.

Table 1. Data elements $(n=112)$ that were identified for the nursing assessment of cancer survivors

\begin{tabular}{|c|c|c|c|c|}
\hline \multicolumn{5}{|c|}{ Data elements $(n=112)$} \\
\hline $\begin{array}{l}\text { Physical domain } \\
\qquad(\mathrm{n}=60)\end{array}$ & $\begin{array}{l}\text { Psychological domain } \\
\qquad(n=37)\end{array}$ & $\begin{array}{l}\text { Social domain } \\
(n=5)\end{array}$ & $\begin{array}{l}\text { Cognitive domain } \\
\qquad(n=11)\end{array}$ & $\begin{array}{l}\text { Spiritual domain } \\
\qquad(\mathrm{n}=5)\end{array}$ \\
\hline $\mathrm{n}=60$ & \multicolumn{4}{|c|}{$\mathrm{n}=2$} \\
\hline Agnosia, alopecia, anorexia, & \multicolumn{4}{|c|}{ Posttraumatic growth, adaptation } \\
\hline appetite, breast engorge- & $\mathrm{n}=35$ & $\mathrm{n}=3$ & $\mathrm{n}=9$ & $\mathrm{n}=3$ \\
\hline ment, cancer fatigue, & Anger, anxiety, cognitive im- & Communication, & Decision making difficulty, & Spiritual interests, \\
\hline caregiver exhaustion, & pairment, concern, coping, & loneliness, distrust & lack of judgment, body & doubt, apathy \\
\hline chemotherapy nausea, & decreased concentration, & & image, responsibility, will, & \\
\hline chemotherapy vomit- & decreased sense of real- & & insight, knowledge, memo- & \\
\hline ing, chock, constipation, & ity, demotivation, denial, & & ry, lack of knowledge & \\
\hline cough, daily activity, death, & dependence, depression, & & & \\
\hline denture, desire to urina- & disappointment, disgust, & & & \\
\hline tion, diarrhea, discharge, & dissatisfaction, distress, & & & \\
\hline discomfort, dizziness, & emotion, fear, frustration, & & & \\
\hline double vision, drainage, dry & grief, guilty, helplessness, & & & \\
\hline mucous membrane, dry & hopelessness, indifference, & & & \\
\hline skin, dyspepsia, dysphagia, & mourning, perseverance, & & & \\
\hline dyspnea, dysuria, eating & personality change, pessi- & & & \\
\hline habit, electrolyte imbalance, & mistic, powerlessness, preju- & & & \\
\hline emaciation, encopresis, en- & dice, projection, restlessness, & & & \\
\hline uresis, exopthalmos, facial & sadness, suicidal intention, & & & \\
\hline flushing, faint, falling, fever, & unpleasant, acceptance & & & \\
\hline flatulence, fluid intake, food & & & & \\
\hline intake, foreign substance, & & & & \\
\hline gynecomstia, heart burn, & & & & \\
\hline impaired urinary elimina- & & & & \\
\hline tion, lethargy, nausea, pain, & & & & \\
\hline paralysis, sexual dysfunc- & & & & \\
\hline tion, skin integrity, stomati- & & & & \\
\hline tis, tingling, tissue integrity, & & & & \\
\hline urinary retention, urina- & & & & \\
\hline tion, vomiting, weight loss, & & & & \\
\hline foul odor, insomnia & & & & \\
\hline
\end{tabular}


Fifty four patients (55.1\%) were GI cancer (i.e., stomach, colon), twenty nine (29.6\%) were breast cancer, ten $(10.2 \%)$ were gynecological cancer, and five (5.1\%) were other types of cancer (i.e., lung, liver). In total, 112 data elements were identified. Forty-four data elements (39.3\%) were extracted by extracting key concepts from clinical nursing statement. Sixty four data elements (57.1\%) were identified from literature review, and four data elements (3.6\%) were identified from the experts' evaluation. Table 1 presents the final data elements that we identified for the nursing assessment of cancer survivors. Sixty data elements were classified as physical domains, 37 as psychological domains, 10 as cognitive domains, 4 as social domains, and 4 as spiritual domains. Posttraumatic growth and adaptation cannot be classified as one domain because it has multiple characteristics with psychological, social, cognitive, and spiritual aspects. Data elements were also classified into three groups based on the direction of judgment (i.e., positive, negative, or neutral) (Table 2).

\section{Defining Critical Attributes, Value Sets, Cardinalities, and Data Types}

Table 3 lists the critical attributes that we identified to express the data elements, and the frequency of these critical attributes used therein. In total, 29 critical attributes were identified. Occurrence, progression, duration, severity, and frequency appeared in more than $60 \%$ of the data elements, while interpretation, onset, and anatomical site appeared in about $20 \%$. Example value sets of these attributes are presented in Table 4 . In total, 102 value sets were identified. In the model development process, we identified two data elements that are used interchangeably in nursing practice with different critical attributes or value sets. Examples are "discharge" and "drainage," and "weight loss" and "emaciation."

Table 2. The grouping of data elements of nursing assessment for cancer survivors based on the direction of meaning judgment of data elements

\begin{tabular}{|c|c|c|}
\hline $\begin{array}{l}\text { Data elements with negative } \\
\text { judgment position }(n=95)\end{array}$ & $\begin{array}{l}\text { Data elements with positive } \\
\text { judgment position }(n=4)\end{array}$ & $\begin{array}{l}\text { Data elements with neutral } \\
\text { judgment position }(n=13)\end{array}$ \\
\hline $\begin{array}{l}\text { Agnosia, alopecia, anger, anorexia, anxiety, apa- } \\
\text { thy, breast engorgement, cancer fatigue, caregiver } \\
\text { exhaustion, chemotherapy nausea, chemotherapy } \\
\text { vomiting, chock, cognitive impairment, concern, } \\
\text { constipation, coping, cough, decision making dif- } \\
\text { ficulty, decreased concentration, decreased sense of } \\
\text { reality, demotivation, denial, denture, dependence, } \\
\text { depression, diarrhea, disappointment, discharge, } \\
\text { discomfort, disgust, dissatisfaction, distress, dis- } \\
\text { trust, disturbed body image, dizziness, double } \\
\text { vision, doubt, drainage, dry mucous membrane, } \\
\text { dry skin, dyspepsia, dysphagia, dyspnea, dysuria, } \\
\text { electrolyte imbalance, emaciation, encopresis, } \\
\text { enuresis, exopthalmos, facial flushing, faint, fear, } \\
\text { fever, flatulence, fluid intake, food intake, foreign } \\
\text { substance, foul odor, frustration, grief, guilty } \\
\text { conscience, gynecomstia, heart burn, helpless- } \\
\text { ness, hopelessness, impaired urinary elimination, } \\
\text { indifference, insomnia, lack of judgment, lack of } \\
\text { knowledge, lethargy, loneliness, mourning, nausea, } \\
\text { pain, paralysis, personality change, pessimistic, } \\
\text { powerlessness, prejudice, projection, restlessness, } \\
\text { sadness, sexual dysfunction, skin integrity, stoma- } \\
\text { titis, suicidal intention, tingling, tissue integrity, un- } \\
\text { falling, death }\end{array}$ & $\begin{array}{l}\text { Spiritual interests, posttraumatic } \\
\text { growth, adaptation, acceptance }\end{array}$ & $\begin{array}{l}\text { Desire to urination, appetite, urina- } \\
\text { tion, eating habit, daily activity, } \\
\text { emotion, memory, insight, knowl- } \\
\text { edge, communication, perseverance, } \\
\text { responsibility, will }\end{array}$ \\
\hline
\end{tabular}


Table 3. The critical attributes that we identified to express the data elements, and the frequency of these critical attributes used therein

\begin{tabular}{|c|c|c|}
\hline No. & Critical attributes & $\begin{array}{c}\text { Frequency of use in } \\
\text { data elements }\end{array}$ \\
\hline 1 & Occurrence & $96(85.7)$ \\
\hline 2 & Progression & $75(67.0)$ \\
\hline 3 & Duration & $74(66.1)$ \\
\hline 4 & Severity & $74(66.1)$ \\
\hline 5 & Frequency & $71(63.4)$ \\
\hline 6 & Interpretation & $22(19.6)$ \\
\hline 7 & Onset & $20(17.9)$ \\
\hline 8 & Anatomical site & $17(15.2)$ \\
\hline 9 & Type & $14(12.5)$ \\
\hline 10 & Quantity (numerical quantity) & $12(10.7)$ \\
\hline 11 & Time sequence & $8(7.1)$ \\
\hline 12 & Color & $8(7.1)$ \\
\hline 13 & Character & $6(5.4)$ \\
\hline 14 & Odor & $4(3.6)$ \\
\hline 15 & Target & $4(3.6)$ \\
\hline 16 & Device & $3(2.7)$ \\
\hline 17 & Substance & $3(2.7)$ \\
\hline 18 & Place & $2(1.8)$ \\
\hline 19 & Number of lesions & $2(1.8)$ \\
\hline 20 & Size & $2(1.8)$ \\
\hline 21 & Regularity & $2(1.8)$ \\
\hline 22 & Extent & $1(0.9)$ \\
\hline 23 & Method & $1(0.9)$ \\
\hline 24 & Ventilation & $1(0.9)$ \\
\hline 25 & Salty & $1(0.9)$ \\
\hline 26 & Turbidity & $1(0.9)$ \\
\hline 27 & Laterality & $1(0.9)$ \\
\hline 28 & Sound & $1(0.9)$ \\
\hline 29 & Radiation & $1(0.9)$ \\
\hline
\end{tabular}

Values are presented as number (\%).

The data element "drainage" has the critical attribute "device" with a value such as "Hemovac" or "rubber." However, the data element "discharge" does not have a critical attribute "device." "Weight loss" and "emaciation" differ with regard to the critical attribute "severity". The data element "emaciation" does not require the critical attribute "severity" because it means excessive leanness. This shows that data modeling improves the accuracy of nurses' documentation of data.

The cardinalities of the attributes, that is whether the at- tribute was optional or mandatory, were defined. The data type of each attribute was classified based on the HL7 data type list [17]. For example, the data type of precise numbers that are the result of counting and enumerating (e.g., -1, 0, and 3398129) is "integer number", and that of "quantities," which are measured, or computed from other real numbers (e.g., 56.3 and 165.5), is "real number," that of coding as order form (i.e., $0=$ rarely, $1=$ sometimes, $2=$ often, and $3=$ always) is "coded ordinal," that of coding as text form (i.e., localized and generalized) is "coded text," that of recording freely as text form is "text", and that of date and time (i.e., yyyymmddhhmm) is "date \& time."

\section{Constructing Data Models}

Using the 112 data elements, 29 critical attributes, 102 value sets, and 6 data types, we developed 112 data models. Table 5 presents a representative example of a data model for pain. The critical attributes of pain had the following value sets:

- Severity - absent, tolerable, mild, moderate, and severe, or from 0 (no pain) to 10 (very severe pain) on a visual analog scale.

- Progression - acute and chronic.

- Duration - seconds, minutes, and hours (over which the pain persists).

- Frequency - very rarely, sometimes, often, and always.

- Onset - gradual, sudden, and intermittent.

- Time sequence - intermittent, continuous, and waxing and waning.

- Regularity - regular and irregular.

- Occurrence - yyyymmddhhmm.

- Anatomical site - free text.

- Characteristic - prick, ache, burn, throb, dull, and sharp.

- Radiation - yes and no.

The data type of severity and frequency is "coded ordinal," that of progression, onset, time sequence, regularity, characteristic, and radiation is "coded text," that of occurrence is "date \& time", and that of anatomical site is "text." Cardinality determined "severity" as mandatory (Table 5).

We grouped together data models with the same critical attributes. 57 groups of data models in accordance with combinations of attributes were made; for example, distress, sadness, and loneliness belong to the same group. Data models for these data elements have the same critical attributes, duration, frequency, occurrence, progression, and severity (Table 6).

\section{Validation of Data Models}

Some domain experts suggested that several cardinalities of the data models be revised. One of the domain experts 
Table 4. Example value sets of the critical attributes

\begin{tabular}{|c|c|}
\hline Critical attributes & Example value sets $(n=102)$ \\
\hline Anatomical site $(\mathrm{n}=14)$ & $\begin{array}{l}\text { [right breast | left breast | both], [oral | right eye | left eye | both eye | nasal | right ear | left ear | both ear } \\
\text { | neck | skin], [chest | back | leg | arm], [nipple | infection or inflammation site | vagina | eye | nose | cer- } \\
\text { vix], [fingers | hands | feet | arms| legs | wrist], [nail | lip | skin | tongue], [eyelid | breathing passage | di- } \\
\text { gestive passage | genital tract], [fingers | hands | feet | arms | legs], [cheeks | gums | tongue | lips | throat } \\
\text { roof of mouth | floor of the mouth], [right eye | left eye | both], [sacrum | elbows | knees | ankles], [vaginal } \\
\text { | oral | eye | ear | nasal | foot | axilla | hair | whole body], [mandibular arch | the maxillary arch | both], } \\
\text { [free text] }\end{array}$ \\
\hline Character $(n=7)$ & $\begin{array}{l}\text { [serous } \mid \text { sanguineous } \mid \text { mucosanguineous } \mid \text { mucoserous } \mid \text { mucous } \mid \text { purulent], [variable } \mid \text { unchanging } \mid \\
\text { horizontal vertical } \mid \text { oblique], [fatty (mucose) } \mid \text { hard } \mid \text { loose], [Kussmaul } \mid \text { Biot } \mid \text { sighing } \mid \text { atasic respira- } \\
\text { tion | Cheyen-Stokes } \mid \text { apneusi], [serous } \mid \text { sanguineous } \mid \text { mucosanguineous } \mid \text { mucoserous } \mid \text { mucous } \mid \\
\text { purulent | mucopurulent } \mid \text { watery } \mid \text { serosanguineous], [dry | moist } \mid \text { productive], [prick | ache | burn | } \\
\text { throb | dull } \mid \text { sharp] }\end{array}$ \\
\hline Color $(n=6)$ & 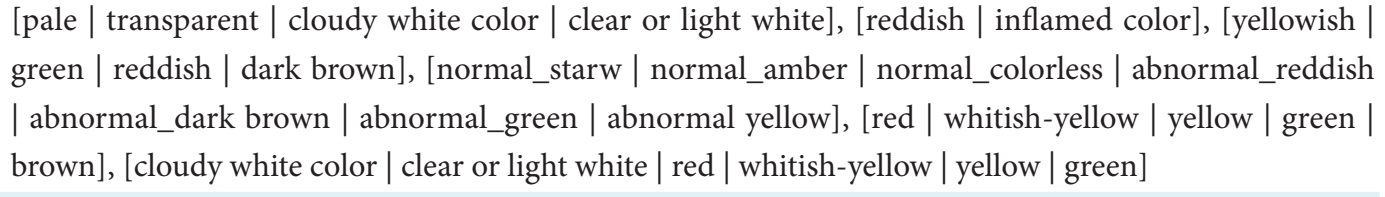 \\
\hline Device $(n=2)$ & [hemovac | J-P | foley], [intermittent catheterization | continuous catheterization] \\
\hline Duration $(n=3)$ & [day $\mid$ week $\mid$ month], [hour $\mid$ day $\mid$ week $\mid$ month], [minute $\mid$ hour $\mid$ day $\mid$ week $\mid$ month] \\
\hline Frequency $(\mathrm{n}=5)$ & $\begin{array}{l}\text { [less than one meal a day } \mid \text { one meal a day } \mid \text { two meals a day } \mid \text { three meals a day } \mid \text { more than three meals } \\
\text { a day], [very rarely } \mid \text { sometimes } \mid \text { often } \mid \text { always], [__counts/day], [__meals/day], [__ count /minute] }\end{array}$ \\
\hline Extent $(\mathrm{n}=1)$ & [localized | generalized] \\
\hline Interpretation $(\mathrm{n}=14)$ & $\begin{array}{l}\text { [alteration with constipation | no alteration with constipation], [voluntary loss of urine } \mid \text { involuntary } \\
\text { loss of urine], [double vision at near } \mid \text { double vision at far], [alternation with diarrhea } \mid \text { no alteration } \\
\text { with diarrhea], [effective } \mid \text { impaired], [risk } \mid \text { no risk], [defensive } \mid \text { ineffective], [under } \mid \text { adequate } \mid \text { over], } \\
\text { [deficient } \mid \text { adequate } \mid \text { over], [able } \mid \text { unable], [expressed } \mid \text { unexpressed], [disturbed } \mid \text { adequate], [deficient } \\
\text { | adequate], [effective } \mid \text { ineffective] }\end{array}$ \\
\hline Laterality $(\mathrm{n}=1)$ & [asymmetry | symmetry] \\
\hline Method $(\mathrm{n}=1)$ & [face to face | synchronous device | asynchronous device] \\
\hline \multicolumn{2}{|c|}{ Number of lesions $(\mathrm{n}=1)[\ldots$ counts $]$} \\
\hline \multicolumn{2}{|c|}{ Numerical quantity $(\mathrm{n}=2)\left[{ }^{\circ} \mathrm{C}\right],\left[\_\mathrm{kg}\right]$} \\
\hline Occurrence $(\mathrm{n}=2)$ & $\begin{array}{l}\text { [yyyymmddhhmm], [pre-treatment } \mid \text { during treatment } \mid 0-4 \text { hours after treatment } \mid 4-8 \text { hours after } \\
\text { treatment | } 8-12 \text { hours after treatment | } 12-24 \text { hours after treatment } \mid 24 \text { or more hours after treatment } \mid \\
\text { no time more severe than any other] }\end{array}$ \\
\hline Odor $(\mathrm{n}=1)$ & [present $\mid$ absent] \\
\hline Onset $(\mathrm{n}=1)$ & [gradual | sudden | intermittent] \\
\hline Place $(\mathrm{n}=1)$ & [home | ICU | general ward | OPD | death on arrival | hospice•palliative care unit] \\
\hline Progression $(n=2)$ & [acute | chronic], [transient | chronic] \\
\hline Quantity $(\mathrm{n}=3)$ & $\begin{array}{l}\text { [hourly urinary output], [little | a little } \mid \text { moderate } \mid \text { much], [less than usual | like usual | more than } \\
\text { usual] }\end{array}$ \\
\hline Regularity $(\mathrm{n}=1)$ & [regular | irregular] \\
\hline Radiation $(\mathrm{n}=1)$ & [yes $\mid$ no] \\
\hline Salty $(\mathrm{n}=1)$ & [salty | adequate | skinking | sugary | adequate | less sugary] \\
\hline
\end{tabular}


Table 4. Continued

\begin{tabular}{|c|c|}
\hline Critical attributes & Example value sets $(n=102)$ \\
\hline Severity $(\mathrm{n}=8)$ & $\begin{array}{l}\text { [absent } \mid \text { tolerable } \mid \text { mild } \mid \text { moderate } \mid \text { severe], [absent } \mid \text { mild } \mid \text { moderate } \mid \text { severe], [grade } 0|1| 2|3| 4 \text { (from } \\
\text { common terminology criteria for adverse events grade)], [non-blanchable redness } \mid \text { damage to the epi- } \\
\text { dermis } \mid \text { involving the full thickness of the skin ], [non-blanchable redness } \mid \text { damage to the epidermis } \mid \\
\text { involving the full thickness of the skin, or extending into the subcutaneous tissue layer } \mid \text { extending into the } \\
\text { muscle } \mid \text { extending into tendon or even bone } \mid \text { covered with dead cells], [non-blanchable redness } \mid \text { damage } \\
\text { to the epidermis | involving the full thickness of the skin and subcutaneous tissue layer } \mid \text { extending into the } \\
\text { muscle | extending into tendon or even bone } \mid \text { covered with dead cells], [0-100], [0-10] }\end{array}$ \\
\hline Size $(\mathrm{n}=1)$ & [_cm X_cm X_cm] \\
\hline Sound $(\mathrm{n}=1)$ & [brassy | hoarse | wheezy | barking] \\
\hline Substance $(\mathrm{n}=2)$ & [Digested food | clear watery fluid | sticky fluid], [wound discharge | urine | stool | vaginal discharge] \\
\hline Target $(\mathrm{n}=4)$ & $\begin{array}{l}\text { [diagnosis } \mid \text { disease stage], [diagnosis } \mid \text { healthy behavior], [patient } \mid \text { nurse } \mid \text { doctor], [objects } \mid \text { persons } \mid \\
\text { sounds } \mid \text { shapes } \mid \text { smells] }\end{array}$ \\
\hline Time sequence $(\mathrm{n}=2)$ & [continuous | intermittent], [intermittent | continuous | waxing and waning] \\
\hline Turbidity $(\mathrm{n}=1)$ & [bubble mixed | turbid] \\
\hline Type $(\mathrm{n}=13)$ & 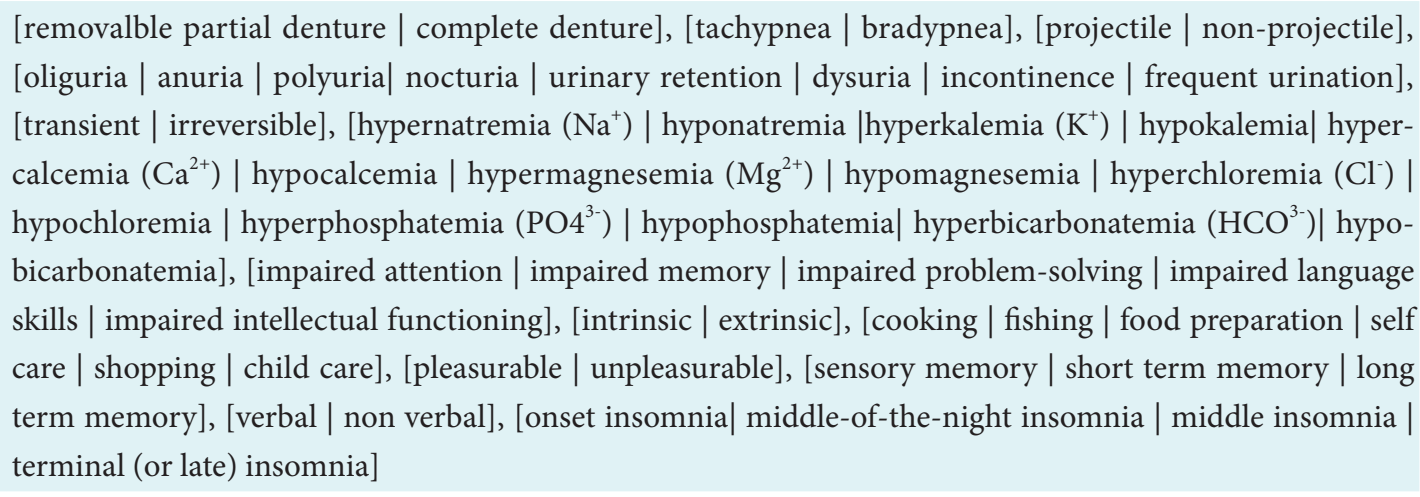 \\
\hline Ventilation $(\mathrm{n}=1)$ & [hyperventilation | hypoventilation | apnea] \\
\hline
\end{tabular}

Table 5. A representative example of a data model for pain

\begin{tabular}{|c|c|c|c|c|}
\hline Data element & Critical attribute & Value set & Data type & Cardinality \\
\hline \multirow[t]{11}{*}{ Pain } & Severity & $\begin{array}{l}\text { absent | tolerable | mild | moderate | severe or } 0 \text {-10; visual } \\
\text { analog scale }\end{array}$ & Coded ordinal & Mandatory \\
\hline & Progression & acute $\mid$ chronic & Coded text & Optional \\
\hline & Duration & second $\mid$ minute $\mid$ hour $\mid$ day & Integer number & Optional \\
\hline & Frequency & very rarely | sometimes | often | always & Coded ordinal & Optional \\
\hline & Onset & gradual | sudden | intermittent & Coded text & Optional \\
\hline & Time sequence & intermittent | continuous | waxing and waning & Coded text & Optional \\
\hline & Regularity & regular |irregular & Coded text & Optional \\
\hline & Occurrence & yymmddhhmm & Date\&time & Optional \\
\hline & Anatomical site & free text & Text & Optional \\
\hline & Characteristic & prick | ache $\mid$ burn $\mid$ throb | dull | sharp & Coded text & Optional \\
\hline & Radiation & yes $\mid$ no & Coded text & Optional \\
\hline $\begin{array}{l}\text { Definition from } \\
\text { international } \\
\text { classification for } \\
\text { nursing practice } \\
\text { [Cancer pain] }\end{array}$ & \multicolumn{4}{|c|}{$\begin{array}{l}\text { Concurrently sensations of acute and chronic pain of different levels of intensity associated with invasive } \\
\text { spreading of cancer cells in the body; consequences of cancer treatment including chemotherapy, or condi- } \\
\text { tions related to cancer such as wound pain; cancer pain is usually reported as dull, hurting, aching, frightful } \\
\text { or unbearable pain sensation with attach of intensive sensations of pain accompanied by sleep difficulties, } \\
\text { irritability, depression, suffering, isolation hopelessness and helplessness. }\end{array}$} \\
\hline
\end{tabular}


Table 6. Groups of data models in accordance with combinations of critical attributes

\begin{tabular}{|c|c|c|}
\hline $\begin{array}{l}\text { Group of } \\
\text { data model }\end{array}$ & Data elements & Critical attributes \\
\hline Group 1 & $\begin{array}{l}\text { Distress, sadness, lethargy, loneliness, mourning, } \\
\text { sexual dysfunction, decreased sense of reality, } \\
\text { decreased concentration, concern, anger, anxiety, } \\
\text { apathy, depression, dyspepsia, dysuria, fear,grief, } \\
\text { unpleasant, disgust, distrust, dysphagia, decision } \\
\text { making difficulty, demotivation, denial, dissatis- } \\
\text { faction, caregiver exhaustion, helplessness, hope- } \\
\text { lessness, dependence, powerlessness, projection, } \\
\text { frustration, doubt, indifference, guilty conscience, } \\
\text { disappointment, encopresis }\end{array}$ & Duration, Frequency, Occurrence, Progression, Severity \\
\hline Group 2 & Heart burn, dizziness, facial flushing & $\begin{array}{l}\text { Duration, frequency, occurrence, progression, severity, time se- } \\
\text { quence }\end{array}$ \\
\hline Group 3 & Discomfort, breast engorgement & $\begin{array}{l}\text { Duration, frequency, occurrence, onset, progression, severity, time } \\
\text { sequence, anatomical site }\end{array}$ \\
\hline Group 4 & Pain & $\begin{array}{l}\text { Duration, frequency, occurrence, onset, progression, severity, time } \\
\text { sequence, anatomical site, radiation }\end{array}$ \\
\hline Group 5 & Enuresis & $\begin{array}{l}\text { Duration, frequency, occurrence, onset, progression, severity, } \\
\text { quantity }\end{array}$ \\
\hline Group 6 & Fever & $\begin{array}{l}\text { Duration, frequency, occurrence, onset, progression, time se- } \\
\text { quence, numerical quantity }\end{array}$ \\
\hline Group 7 & Chock & Duration, frequency, severity \\
\hline Group 8 & Dyspnea & $\begin{array}{l}\text { Duration, frequency, occurrence, onset, severity, type, character, } \\
\text { ventilation }\end{array}$ \\
\hline Group 9 & Diarrhea & Duration, frequency, occurrence, onset, progression, severity \\
\hline Group 10 & Discharge & $\begin{array}{l}\text { Duration, frequency, occurrence, anatomical site, quantity, color, } \\
\text { odor, character }\end{array}$ \\
\hline Group 11 & Drainage & $\begin{array}{l}\text { Duration, frequency, occurrence, quantity, anatomical site, odor, } \\
\text { color, character, device }\end{array}$ \\
\hline Group 12 & Cough & $\begin{array}{l}\text { Duration, frequency, occurrence, progression, severity, character, } \\
\text { sound }\end{array}$ \\
\hline Group 13 & Vomiting & $\begin{array}{l}\text { Duration, frequency, occurrence, progression, severity, quantity, } \\
\text { color, substance, type }\end{array}$ \\
\hline Group 14 & Chemotherapy vomiting & $\begin{array}{l}\text { Duration, frequency, occurrence, severity, quantity, color, sub- } \\
\text { stance, type }\end{array}$ \\
\hline Group 15 & Nausea & Duration, frequency, occurrence, progression, severity \\
\hline Group 16 & Chemotherapy nausea & Duration, frequency, occurrence, severity \\
\hline Group 17 & Paralysis & Duration, occurrence, onset, extent, anatomical site, laterality \\
\hline Group 18 & Dry mucous membrane, dry skin, tingling & Duration, occurrence, progression, severity, anatomical site \\
\hline Group 19 & Impaired urinary elimination & Duration, occurrence, onset, progression, severity, type \\
\hline Group 20 & Fatigue & $\begin{array}{l}\text { Duration, occurrence, onset, progression, severity, symptom- } \\
\text { related fatigue }\end{array}$ \\
\hline Group 21 & Urinary retention & $\begin{array}{l}\text { Duration, occurrence, progression, severity, quantity, description, } \\
\text { device, interpretation }\end{array}$ \\
\hline
\end{tabular}


Table 6. Continued

\begin{tabular}{|c|c|c|}
\hline $\begin{array}{l}\text { Group of } \\
\text { data model }\end{array}$ & Data elements & Critical attributes \\
\hline Group 22 & Stomatitis & Duration, occurrence, progression, severity, anatomical site \\
\hline Group 23 & Alopecia, anorexia & Duration, occurrence, progression, severity \\
\hline Group 24 & Weight loss & Duration, occurrence, onset, severity, numerical quantity \\
\hline Group 25 & Emaciation & Duration, occurrence, onset, numerical quantity \\
\hline Group 26 & Double vision & $\begin{array}{l}\text { Duration, occurrence, onset, severity, time sequence, interpre- } \\
\text { tation, character, anatomical site }\end{array}$ \\
\hline Group 27 & Agnosia & Duration, occurrence, onset, progression, severity, type, target \\
\hline Group 28 & Constipation & $\begin{array}{l}\text { Duration, occurrence, onset, progression, severity, interpreta- } \\
\text { tion }\end{array}$ \\
\hline Group 29 & Skin integrity & $\begin{array}{l}\text { Duration, occurrence, severity, anatomical site, color, inter- } \\
\text { pretation, number of lesions, size }\end{array}$ \\
\hline Group 30 & Tissue integrity & $\begin{array}{l}\text { Duration, occurrence, severity, anatomical site, color, inter- } \\
\text { pretation, number of lesions, size }\end{array}$ \\
\hline Group 31 & Insomnia & Duration, frequency, occurrence, progression, severity, type \\
\hline Group 32 & Suicidal intention & Frequency, occurrence, progression \\
\hline Group 33 & Flatulence & Frequency, occurrence, progression, odor \\
\hline Group 34 & Lack of judgment, pessimistic, prejudice & Frequency, occurrence, progression, severity \\
\hline Group 35 & Restlessness & Frequency, occurrence, progression, severity, time sequence \\
\hline Group 36 & Urination & $\begin{array}{l}\text { Frequency, occurrence, numerical quantity, color, device, } \\
\text { odor, interpretation, turbidity }\end{array}$ \\
\hline Group 37 & Faint & Frequency, occurrence, progression \\
\hline Group 38 & Falling & Frequency, occurrence, severity, cause, place \\
\hline Group 39 & Desire to urination & Frequency, interpretation \\
\hline Group 40 & Appetite & Eating frequency, interpretation, description \\
\hline Group 41 & Defecation pattern & Frequency, numerical quantity, color, character, regularity \\
\hline Group 42 & Eating habit & Frequency, quantity, interpretation, regularity, salty \\
\hline Group 43 & Personality change & Occurrence, onset \\
\hline Group 44 & Exopthalmos & Occurrence, onset, severity, anatomical site \\
\hline Group 45 & Electrolyte imbalance & Occurrence, onset, severity, type \\
\hline Group 46 & Spiritual interest, posttraumatic growth & Occurrence, onset \\
\hline Group 47 & Gynecomstia & Occurrence, onset, anatomical site \\
\hline Group 48 & Cognitive impairment, demotivation & Occurrence, progression, severity, type \\
\hline Group 49 & Lack of knowledge & Occurrence, progression, severity \\
\hline Group 50 & Foreign substance & Occurrence, progression, anatomical site \\
\hline Group 51 & Death & Occurrence, cause, place \\
\hline Group 52 & Foul odor & Occurrence, progression, severity, anatomical site, substance \\
\hline Group 53 & $\begin{array}{l}\text { Coping, fluid intake, food intake, perseverance, re- } \\
\text { sponsibility, will, body image }\end{array}$ & Interpretation \\
\hline Group 54 & Daily activity, emotion, memory & Type, interpretation \\
\hline Group 55 & Insight, knowledge & Target, interpretation \\
\hline Group 56 & Denture & Anatomical site, type \\
\hline Group 57 & Communication & Type, interpretation, target, method \\
\hline
\end{tabular}


Table 7. External validation: responses of domain experts.

\begin{tabular}{|c|c|c|c|c|c|}
\hline Items $(n=1,232)$ & $\begin{array}{l}\text { Strongly } \\
\text { agree }\end{array}$ & Agree & $\begin{array}{l}\text { Neither agree } \\
\text { nor disagree }\end{array}$ & Disagree & $\begin{array}{l}\text { Strongly } \\
\text { disagree }\end{array}$ \\
\hline \multicolumn{6}{|l|}{ Clinical relevancy } \\
\hline Is the data element clinically meaningful? & $152(12.3)$ & $712(57.8)$ & $338(27.4)$ & $25(2.0)$ & $5(0.4)$ \\
\hline $\begin{array}{l}\text { Are the critical attributes of the data element clinically } \\
\text { meaningful? }\end{array}$ & $176(14.3)$ & $747(60.6)$ & $287(23.3)$ & $22(1.8)$ & $0(0.0)$ \\
\hline \multicolumn{6}{|l|}{ Usefulness } \\
\hline Is the data element useful for assessing cancer patients? & $192(15.6)$ & $805(65.3)$ & $229(18.6)$ & $6(0.5)$ & $0(0.0)$ \\
\hline $\begin{array}{l}\text { Are the critical attributes useful for expressing the data } \\
\text { element? }\end{array}$ & $253(20.5)$ & $818(66.4)$ & $156(12.7)$ & $4(0.3)$ & $1(0.1)$ \\
\hline \multicolumn{6}{|l|}{ Reusability } \\
\hline Is the data model reusable? & $176(14.3)$ & $824(66.9)$ & $208(16.9)$ & $23(1.9)$ & $1(0.1)$ \\
\hline \multicolumn{6}{|l|}{ Non-ambiguity } \\
\hline Is the data model clear? & $250(20.3)$ & $842(68.3)$ & $118(9.6)$ & $22(1.8)$ & $0(0.0)$ \\
\hline \multicolumn{6}{|l|}{ Comprehensiveness } \\
\hline $\begin{array}{l}\text { Are the data elements sufficiently comprehensive to } \\
\text { assess cancer survivors? }^{\mathrm{a}}(\mathrm{n}=11)\end{array}$ & $3(27.3)$ & $6(54.6)$ & $1(9.1)$ & $1(9.1)$ & $0(0.0)$ \\
\hline Are the critical attributes comprehensive in the data model? & $123(10.0)$ & $981(79.6)$ & $94(7.6)$ & $31(2.5)$ & $3(0.2)$ \\
\hline Is the value set comprehensive in a critical attribute? & $220(17.9)$ & $910(73.9)$ & $55(4.5)$ & $41(3.3)$ & $6(0.5)$ \\
\hline \multicolumn{6}{|l|}{ Non-redundancy } \\
\hline Are there any redundant critical attributes in the data model? & $0(0.0)$ & $0(0.0)$ & $0(0.0)$ & $1,035(84.0)$ & $197(16.0)$ \\
\hline Are there any redundant values in the critical attributes? & $0(0.0)$ & $0(0.0)$ & $0(0.0)$ & $845(68.6)$ & $387(31.4)$ \\
\hline
\end{tabular}

Values are presented as number (\%).

aThis question was asked to 11 external domain experts about 112 data models. Except for this case, total answer is 1,232.

indicated the value set of "absent, tolerable, mild, moderate and severe" of 'severity' critical attribute in the data element of pain gave a limited illustration. In the clinical practice, most pain is assessed using a 0-10 scale which should allow a more detailed analysis. Based on this, the value set of $0-10$ visual analogue scale was added as one of value sets of severity critical attributes of pain model.

Expert panel of clinicians suggested developing new data models of body image, posttraumatic growth, insight, and knowledge. More than $80 \%$ of expert panel of clinicians rated the 112 data models using a response of "strongly agree" or "agree" to the questions of "usefulness," "reusability," "nonambiguity," "comprehensiveness," and "nonredundancy." For "clinical relevancy", 70.1\% responded "strongly agree" or "agree" to the question "Is the data element clinically meaningful?" (Table 7).

\section{Discussion}

Healthcare professionals of various types in a variety of hospitals need to follow up cancer patients to monitor or prevent recurrences or secondary cancer even after successful treatment of the primary cancer. The ability to share cancerrelated information among many healthcare professionals and different hospitals is a prerequisite for maintaining the quality of cancer care. In order to share and exchange clinical data, it should be semantically interoperable. One way of ensuring semantic interoperability is to develop data models of the clinical data.

Concepts should be analyzed to develop data models. In the area of oncology nursing, concept analyses have been limited to specific concepts such as "cancer symptom cluster" [24], "symptom experience" [25], "cancer survivorship" [26], "psychological distress" [27], "suffering" [28], and "symptom disclosure" [29]. Common signs and symptoms of cancer survivors, and survivorship issues related to nursing assess- 
ment have not been analyzed to date. From this background, we developed 112 data models for the nursing assessment of cancer survivors using concept analysis by analyzing nursing documentations, reviewing the literature, and consulting nurse experts.

We extracted data elements describing physical problems from nursing documents and describing psychological and social problems from a literature review of articles, which are usually overlooked on nursing practice [30]. Similarly, we extracted data elements describing positive judgments (i.e., spiritual interests and posttraumatic growth) from the literature review and as a result of the suggestions of our external domain experts. Using a data model describing positive spiritual or psychological changes makes it possible for nurses to document positive outcomes in nursing practice.

The data models developed in this study have cancer-specific attributes. For example, fatigue in a healthy population can be described as "acute fatigue," which can be relieved by sleep and rest. However, cancer-related fatigue can only be chronic because it is present over a long period of time and cannot be not completely relieved by sleep and rest [1]. Thus, the data model for fatigue in healthy people has an attribute "progression" to describe acute and chronic fatigue; however, the equivalent model for cancer-related fatigue does not need the attribute "progression" because cancer-related fatigue is always chronic [1].

The data models, data types and cardinalities of the critical attributes developed in the present study were found to be valid. Even though we developed questions to check the face validity of the models based on earlier studies [19-23], the reliability and validity of the questionnaires were not evaluated vigorously. Thus, we would like to suggest a further study to evaluate the reliability and validity of the questions used to test face validity. Although we evaluated the applicability of the model indirectly by having nurses with clinical experience in oncology evaluate the model, we suggest a further study to test the direct applicability of the model to oncology nursing practice.

Nursing statements used in the current electronic nursing record (ENR) system in Korea comprise simple phrases that describe the judgment on a key data element (e.g., pulse deficit and severe numbness). However, using a data model with critical attributes, value sets, data types, and cardinalities for the ENR allows the key data elements to be documented in more detail and consistently. This will improve the quality of nursing records and, in turn, make the nursing record reusable for research and future practice.

In this study, we were able to develop data models by connecting data elements, critical attributes, and value sets and specifying the data types and cardinalities of the critical attributes. Data models can be used in ENR or EHR systems. The outcomes of this study will contribute to standardized nursing assessment for cancer survivors and improve use of data in clinical practice and research.

\section{Conflict of Interest}

No potential conflict of interest relevant to this article was reported.

\section{Acknowledgements}

This research was supported by Basic Science Research Program through the National Research Foundation of Korea (NRF) funded by the Ministry of Education, Science and Technology (No. 2009-0074695 and No. 2010-0010468).

\section{References}

1. American Cancer Society. Cancer facts \& figures 2008. Atlanta: American Cancer Society; 2008. p57.

2. Twombly R. What's in a name: who is a cancer survivor? J Natl Cancer Inst 2004; 96: 1414-1415.

3. Centers for Disease Control and Prevention (CDC). Cancer survivorship: United States, 1971-2001. MMWR Morb Mortal Wkly Rep 2004; 53: 526-529.

4. Feuerstein, M, Findley P. The cancer survivor's guide: the essential handbook to life after cancer. New York: Marlowe \& Co; 2006. p1.

5. Whippen D, Deering MJ, Ambinder EP. Advancing high-quality cancer care: cancer biomedical informatics grid supports personalized medicine and the electronic health record. J Oncol Pract 2007; 3: 208-211.

6. Graybeal, J. Achieving semantic interoperability. The MMI guides- navigating the world of marine metadata. Marine Metadata Initiative; 2009 [cited at 2011 Mar 15]. Available from: http://marinemetadata.org/guides/vocabs/cvchooseimplement/cvsemint.

7. Hovenga E, Garde S, Heard S. Nursing constraint models for electronic health records: a vision for domain knowledge governance. Int J Med Inform 2005; 74: 886898.

8. Beale T. Archetypes: constraint-based domain models for future-proof information systems. In: Baclawsk, K. Kilov H, eds. Eleventh OOPSLA workshop on behavioral semantics: serving the customer. Boston: Northeastern University; 2002: p16-32.

9. Goossen WT. Using detailed clinical models to bridge 
the gap between clinicians and HIT. Stud Health Technol Inform 2008; 141: 3-10.

10. Huff SM. The GE-intermountain healthcare alliance: a new paradigm for EHR dev elopment. In: Proceeding of the 4th EHR Symposium; 2009; Seoul, KR. p14-28.

11. Kim Y, Huff SM, Ahn SJ, Cho KH, Koh YT. Clinical contents medol: definition, methods, and practical use. In: Proceedings of the 6th Asia Pacific Association for Medical Informatics (APAMI); 2009 Nov 22-24; Hiroshima, JP. pW-03.

12. Johnson SB. Generic data modeling for clinical repositories. J Am Med Inform Assoc 1996; 3: 328-339.

13. Walker LO, Avant KC. Strategies for theory construction in nursing. Norwlk, CT: Appleton \& Lange; 1988.

14. Matteson P, Hawkins JW. Concept analysis of decision making. Nurs Forum 1990; 25: 4-10.

15. Hawks JH. Power: a concept analysis. J Adv Nurs 1991; 16: 754-762.

16. Henneman EA, Lee JL, Cohen JI. Collaboration: a concept analysis. J Adv Nurs 1995; 21: 103-109.

17. Schadow G, Brion P, McKenzie L, Grieve G, Pratt D. HL7 version 3 standard. Data types - abstract specification, release 1. Health Level Seven ${ }^{\mathrm{TM}}$ Inc. ; 2004 [cited at 2010 Mar 15]. Available from: http://www.hl7.org/ v3ballot/html/infrastructure/datatypes/datatypes.html.

18. Dolin RH, Alschuler L, Boyer S, Beebe C, Behlen FM, Biron PV, Shabo Shvo A. HL7 clinical document architecture: Release 2. J Am Med Inform Assoc 2006; 13: 3039.

19. Chute CG, Cohn SP, Campbell JR. A framework for comprehensive health terminology systems in the United States: development guidelines, criteria for selection, and public policy implications. ANSI healthcare informatics standards board vocabulary working group and the computer-based patient records institute working group on codes and structures. J Am Med Inform Assoc
1998; 5: 503-510.

20. Bakhshi-Raiez F, Cornet R, de Keizer NF. Development and application of a framework for maintenance of medical terminological systems. J Am Med Inform Assoc 2008; 15: 687-700.

21. Kim TY, Coenen A, Hardiker N. A quality improvement model for healthcare terminologies. J Biomed Inform 2010; 43: 1036-1043.

22. Cimino JJ, Clayton PD, Hripcsak G, Johnson SB. Knowledge-based approaches to the maintenance of a large controlled medical terminology. J Am Med Inform Assoc 1994; 1: 35-50.

23. Campbell JR, Carpenter P, Sneiderman C, Cohn S, Chute CG, Warren J. Phase II evaluation of clinical coding schemes: completeness, taxonomy, mapping, definitions, and clarity: CPRI work group on codes and structures. J Am Med Inform Assoc 1997; 4: 238-251.

24. Kim HJ, McGuire DB, Tulman L, Barsevick AM. Symptom clusters: concept analysis and clinical implications for cancer nursing. Cancer Nurs 2005; 28: 270-282.

25. Armstrong TS. Symptoms experience: a concept analysis. Oncol Nurs Forum 2003; 30: 601-606.

26. Doyle N. Cancer survivorship: evolutionary concept analysis. J Adv Nurs 2008; 62: 499-509.

27. Ridner SH. Psychological distress: concept analysis. J Adv Nurs 2004; 45: 536-545.

28. Fochtman D. The concept of suffering in children and adolescents with cancer. J Pediatr Oncol Nurs 2006; 23: 92-102.

29. Sun Y, Knobf MT. Concept analysis of symptom disclosure in the context of cancer. ANS Adv Nurs Sci 2008; 31: 332-341.

30. Wen KY, Gustafson DH. Needs assessment for cancer patients and their families. Health Qual Life Outcomes 2004; 2 : 11. 